Recepción: 20 / 04 / 2017

Aceptación: 20 / 05 / 2017

Publicación: 15 / 06 / 2017

Ciencias Económicas y Empresariales

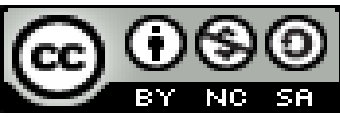

Artículo de Investigación

\title{
Evidencia de la disminución de la desigualdad y su relación con el crecimiento económico en América Latina
}

Evidence of the decline in inequality and its relation to economic growth in Latin America

\section{Evidência de declínio da desigualdade e sua relação com o crescimento econômico} na América Latina

Ángel G. Salazar-Bustos ${ }^{\text {I }}$ angel.salazarb@ug.edu.ec

Ligia J. Fariño-Maldonado II ligia.farinom@ug.edu.ec

Natalia G. Andrade-Moreira ${ }^{\text {III }}$ natalia.andradem@ug.edu.ec

Correspondencia: angel.salazarb@ug.edu.ec

I. Economista; Magister en Finanzas y Proyectos Corporativos; Diploma Superior en Diseño Curricular por Competencias; Diploma Superior en Autoevaluación con Fines de Acreditación; docente Titular de la Facultad de Ciencias Económicas de la Universidad de Guayaquil, Guayaquil, Ecuador.

II. Economista; Magister en Tributación y Finanzas; Diploma Superior en Tributación; Diploma Superior en Pedagogía; docente Titular de la Facultad de Ciencias Económicas de la Universidad de Guayaquil, Guayaquil, Ecuador.

III. Economista; Magister en Negocios Internacionales y Gestión de Comercio Exterior; Diploma Superior en Economía Internacional; docente Titular de la Facultad de Ciencias Económicas de la Universidad de Guayaquil, Guayaquil, Ecuador. 
Evidencia de la disminución de la desigualdad y su relación con el crecimiento económico en América Latina

\section{Resumen}

El presente artículo hace una prueba de la hipótesis de la Curva Kuznets la cual relaciona el crecimiento económico, medido por el PIB per cápita y la desigualdad, medida por el coeficiente GINI, para una muestra de 12 países de América Latina durante el periodo 2000-2012. Además del PIB per cápita se incluyó variables de control como el gasto social, el gasto en educación, el índice de efectividad gubernamental, la recaudación fiscal y la tasa de desempleo, la metodología usada en el presente estudio es de datos de panel. Latinoamérica es considerada una de las regiones más desiguales del planeta, sin embargo en los últimos años esta desigualdad ha estado disminuyendo y al mismo tiempo ha tenido un importante crecimiento económico. Los resultados sugieren que a medida que la región se desarrolle económicamente la desigualdad tiende a disminuir. El gasto social, donde está incluido el de la educación parece ser una buena forma de disminuir esta desigualdad. Cabe mencionar que en el periodo de estudio han existido Gobiernos con una tendencia socialista que se han enfocado en la igualdad social con respecto a los Gobiernos anteriores, lo cual se considera una pieza fundamental para que se pueda cumplir la hipótesis.

Palabras claves: Crecimiento económico; gasto social; igualdad social. 


\begin{abstract}
The present article tests the hypothesis of the Kuznets Curve which relates economic growth, measured by GDP per capita and inequality, measured by the GINI coefficient, for a sample of 12 Latin American countries during the period 2000- 2012. In addition to GDP per capita, we included control variables such as social spending, education spending, government effectiveness index, tax collection and unemployment rate, the methodology used in this study is panel data. Latin America is considered one of the most unequal regions of the planet, however in recent years this inequality has been declining and at the same time has had significant economic growth. The results suggest that as the region develops economically inequality tends to decline. Social spending, which includes education, seems to be a good way to reduce this inequality. It should be mentioned that in the period of study there have been governments with a socialist tendency that have focused on social equality with respect to the previous governments, which is considered a fundamental piece so that the hypothesis can be fulfilled.
\end{abstract}

Key words: economic growth; social spending; social equality. 
Evidencia de la disminución de la desigualdad y su relación con el crecimiento económico en América Latina

\section{Resumo}

Este artigo faz um teste da curva de Kuznets hipótese que relaciona o crescimento econômico medido pelo PIB per capita e da desigualdade, medida pelo coeficiente de GINI para uma amostra de 12 países latino-americanos durante o período de 2000 de 2012. PIB per capita, além de controlar variáveis como o gasto social, gastos com educação, o índice de eficiência do governo, a arrecadação de impostos ea taxa de desemprego foi incluído, a metodologia utilizada neste estudo é de dados em painel. América Latina é considerada uma das regiões mais desiguais do mundo, no entanto, nos últimos anos essa desigualdade tem vindo a diminuir e, ao mesmo tempo, tem tido um crescimento económico significativo. Os resultados sugerem que à medida que a região desenvolve economicamente desigualdade tende a diminuir. o gasto social, que está incluído educação parece ser uma boa maneira de reduzir essa desigualdade. Vale ressaltar que os governos têm existido no período de estudo, com uma tendência socialista têm-se centrado na igualdade social com os governos anteriores, o que é considerado uma peça fundamental para que ele possa atender a hipótese.

Palavras-chave: crescimento econômico; despesas sociais; igualdade social. 


\section{Introducción.}

La pobreza y la desigualdad son fenómenos distintos, pero muy ligados. De forma general, una mayor desigualdad implica mayores niveles de pobreza (Macías, 2014).

Los países latinoamericanos no son los más pobres del mundo, pero presentan una gran disparidad en la distribución de la riqueza y el ingreso. Hasta finales del siglo anterior predominó una tendencia en América Latina: las fases de mayor crecimiento económico estuvieron fuertemente ligadas con mayor desigualdad de la riqueza (Sánchez, 2006). Pero partir del año 2000 hasta el 2012, el crecimiento del PIB per cápita en los países de estudio aumentó en promedio en un 36\%, pasando de $\$ 3818$ a $\$ 5208$ (Gráfico 1). A principios de la década de 2000 en la mayoría de los países de la región se dio un proceso de reducción de la desigualdad de la riqueza. Entre 2000 y 2012 el promedio del índice de GINI cayó aproximadamente un 12\%, de 53.63 a 47.69 en los países seleccionados (Grafico 2). Pero es difícil identificar los factores o determinantes que han provocado esta reducción.

América Latina es una región de contrastes: por una parte hay edificios de primer nivel frente a barrios pobres, hospitales con alta tecnología y otros que no cuentan con recursos, vendedores ambulantes frente a grandes y cuantiosos centros comerciales $\mathrm{Y}$ así muchos más contrastes que plasman a una de las regiones más desiguales del planeta.

Además de esto, los especialistas apuntan que el bajo crecimiento económico que tiene actualmente la región puede aumentar o al menos mantener las distancias entre ricos y pobres. Es así que los Gobiernos con recursos limitados no podrán asegurar que más población tenga acceso a servicios básicos. Este panorama representaría retroceder en los logros sociales alcanzados por la 
Evidencia de la disminución de la desigualdad y su relación con el crecimiento económico en América Latina

región en la última década, y esto debería advertir a los Gobiernos de Latinoamérica (Díaz y Mayorga, 2009).

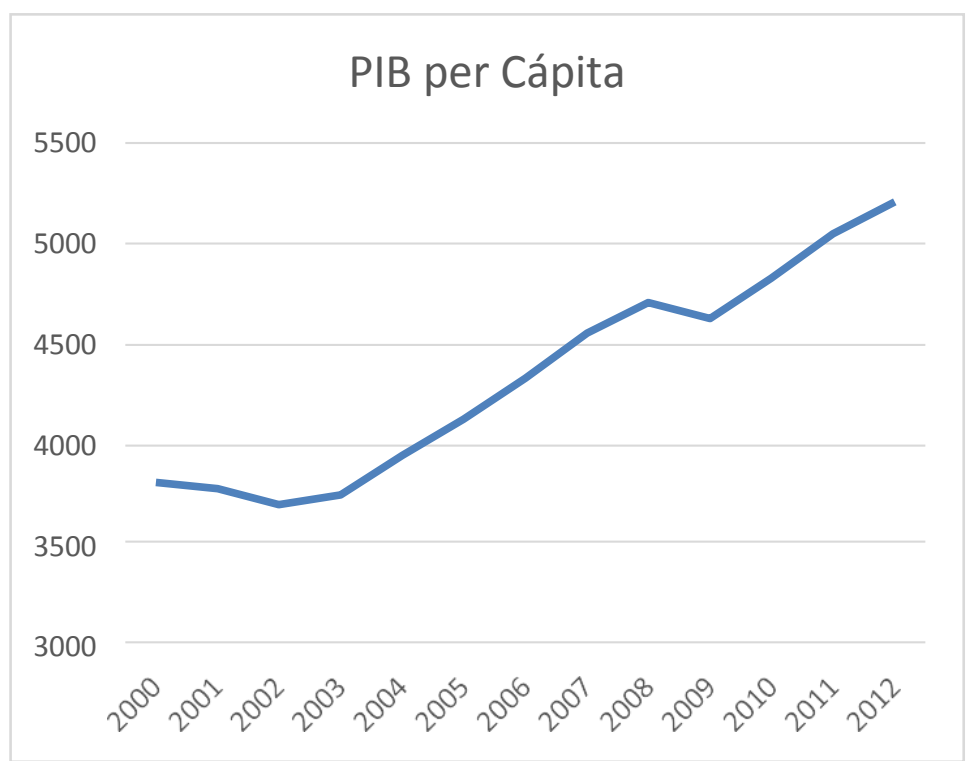

Grafico 1: Crecimiento promedio del PIB per Cápita en América Latina.

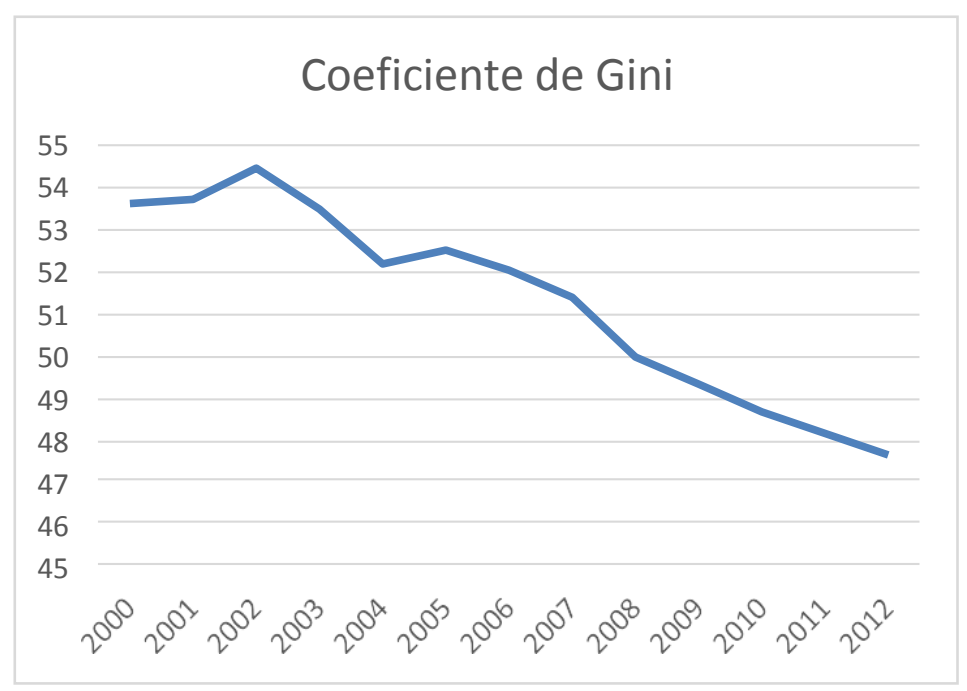

Grafico 2: Coeficiente promedio del GINI en América Latina 


\section{Materiales y métodos.}

En general la relación entre desigualdad y crecimiento se relaciona con la hipótesis de la Curva de Kuznets, la cual establece una relación en forma de U invertida entre la desigualdad y el crecimiento económico (Kuznets, 1955). Se representa en el eje horizontal el ingreso per cápita y en el eje vertical algún indicador de desigualdad, en este caso el coeficiente de GINI.

En el proceso de crecimiento económico de los países inicialmente se aumentaría la desigualdad y disminuiría en etapas posteriores del crecimiento económico. La hipótesis indica que el proceso de crecimiento económico repercute en la distribución del ingreso, forzando a los países a pasar en primera instancia un período de aumento de la desigualdad, donde se espera obtener un crecimiento económico. De esta manera la distribución del ingreso empeoraría en las etapas iniciales del proceso de crecimiento económico, conforme una economía se transforma de agraria en industrial. Posteriormente, la desigualdad disminuiría a medida que la fuerza laboral empleada en la industria se expande mientras que la ocupada en la agricultura decae.

Se decidió tomar la variable coeficiente GINI como la dependiente, la cual será explicada por la variable PIB per cápita junto a otras variables de control. La estrategia empírica aplicada fueron datos de panel, siguiendo las recomendaciones incluidas en Nervole (2000). Además el estudio de Thornton (2010), utilizando datos de panel para una muestra de 96 países concluye que existe una relación en forma de U invertida entre la desigualdad de ingresos y el crecimiento económico.

A diferencia del análisis de corte transversal, los trabajos que utilizan datos de panel consideran variables a lo largo del tiempo para un conjunto de países. Se trata por lo tanto de bases de datos más ricas y útiles para estimaciones de relaciones económicas. Los resultados basados en datos de corte transversal han sido cuestionados por investigaciones basadas en datos de panel, que 
Evidencia de la disminución de la desigualdad y su relación con el crecimiento económico en América Latina

permiten distinguir la trayectoria de cada país, es decir, incluir lo que se denomina efectos fijos por país en las estimaciones. Según Fields y Jakubson (1994), si se introducen los efectos fijos, la curva estimada puede ir de una $\mathrm{U}$ invertida a tener forma de $\mathrm{U}$, y las dos resultan significativas. Para poder controlar por los efectos fijos, es necesario encontrar información confiable para algunos años de los países.

Se tomó una muestra de 15 países de América Latina para el periodo 2000-2012, ya que en estos años se pudo evidenciar un crecimiento económico de los países y al mismo tiempo un decrecimiento del coeficiente GINI. Para todas las estimaciones se utilizó el modelo de efectos fijos, y además se aplicó errores estándar agrupados permitiendo una estimación robusta a heterocedasticidad y autocorrelación.

La tabla 1 muestra las variables consideradas para la investigación junto con una breve descripción y su fuente. La tabla 2 presenta los países analizados con su PIB per cápita y coeficiente GINI para los años 2006 y 2012.

\begin{tabular}{|l|l|l|}
\hline Variables & Descripción & Fuente \\
\hline Coeficiente de GINI & $\begin{array}{l}\text { Indice de desigualdad, } \\
\text { valores entre 0 y1. }\end{array}$ & $\begin{array}{l}\text { Base de datos del Banco } \\
\text { Mundial. }\end{array}$ \\
\hline PIBpc & $\begin{array}{l}\text { PIB per cápita (US\$ a } \\
\text { precios constantes de 2005) }\end{array}$ & $\begin{array}{l}\text { Base de datos del Banco } \\
\text { Mundial. }\end{array}$ \\
\hline Gasto Social & $\begin{array}{l}\text { Gasto público social sobre } \\
\text { el PIB (porcentaje) }\end{array}$ & Cepalstat (ALC) \\
\hline Tasa Desempleo & $\begin{array}{l}\text { Tasa anual media } \\
\text { (porcentaje) }\end{array}$ & $\begin{array}{l}\text { Base de datos del Banco } \\
\text { Mundial. }\end{array}$ \\
\hline IEG & $\begin{array}{l}\text { Indice de Efectividad } \\
\text { Gubernamental }\end{array}$ & $\begin{array}{l}\text { Base de datos Worldwide } \\
\text { Governance Indicators }\end{array}$ \\
\hline Gasto Educación & $\begin{array}{l}\text { Gasto público en educación } \\
\text { sobre PIB (porcentaje) }\end{array}$ & $\begin{array}{l}\text { Cepalstat (ALC) } \\
\text { Recaudación Fiscal }\end{array}$ \\
$\begin{array}{l}\text { Total Ingresos Tributarios } \\
\text { sobre el PIB (porcentaje) }\end{array}$ & Cepalstat (ALC) \\
\hline
\end{tabular}

Tabla 1: descripción de las variables 
Ángel G. Salazar-Bustos; Ligia J. Fariño-Maldonado; Natalia G. Andrade-Moreira

\begin{tabular}{|l|l|l|l|l|}
\hline \multirow{2}{*}{ Países } & \multicolumn{2}{l|l}{ Coeficiente GINI } & \multicolumn{2}{l|}{ PIB per cápita } \\
\cline { 2 - 5 } & $\mathbf{2 0 0 6}$ & $\mathbf{2 0 1 2}$ & $\mathbf{2 0 0 6}$ & $\mathbf{2 0 1 2}$ \\
\hline Argentina & 48,26 & 43,57 & 6050,96 & 7590,07 \\
\hline Bolivia & 56,87 & 46,26 & 1077,97 & 1247,84 \\
\hline Brasil & 55,93 & 53,1 & 4865,18 & 5744,46 \\
\hline Chile & 51,79 & 50,84 & 7978,27 & 9085,24 \\
\hline Colombia & 60,08 & 54,18 & 3567,47 & 4202,14 \\
\hline Costa Rica & 49,31 & 48,6 & 5040,34 & 5683,76 \\
\hline Ecuador & 53,2 & 46,21 & 3102,55 & 3485,07 \\
\hline Honduras & 57,42 & 57,4 & 1470,98 & 1573,07 \\
\hline México & 48,01 & 48,1 & 8162,68 & 8231,70 \\
\hline Panamá & 55,06 & 51,83 & 4967,59 & 6800,08 \\
\hline Paraguay & 53,63 & 52,6 & 1556,15 & 1848,22 \\
\hline Perú & 51,67 & 45,48 & 2883,39 & 3741,74 \\
\hline El Salvador & 45,44 & 42,43 & 2976,51 & 3096,16 \\
\hline Uruguay & 47,2 & 43,37 & 5426,08 & 7204,22 \\
\hline Venezuela & 46,94 & 39,02 & 5873,31 & 6179,26 \\
\hline
\end{tabular}

Tabla 2: países seleccionados

Fuente: Banco Mundial.

En el primer modelo la variable Coeficiente GINI es explicada solamente por el PIB per cápita, debido a que se quiso probar el efecto de la variable de interés sobre la dependiente. Para los siguientes modelos se fueron agregando las distintas variables de control como son gasto social, tasa de desempleo, índice efectividad gubernamental, gasto en educación y recaudación fiscal.

En cuanto a los signos se espera que el coeficiente de PIB per cápita sea negativo debido a que la hipótesis plantea que un incremento del crecimiento económico debería reducir la 
Evidencia de la disminución de la desigualdad y su relación con el crecimiento económico en América Latina

desigualdad, tanto gasto social como educación se espera que sean negativos ya que un mayor gasto de estos debería reducir la desigualdad de un país lo cual está sustentado por estudios previos, así mismo para índice de efectividad gubernamental se espera un coeficiente negativo ya que si se implementan buenas medidas políticas en el país se puede lograr reducir la desigualdad, y por último para tasa de desempleo se espera un coeficiente positivo ya que un mayor desempleo puede provocar un desequilibrio en la distribución de los ingresos.

\section{Resultados.}

\begin{tabular}{ccccc} 
VARIABLES & ESTIMACION 1 & ESTIMACION 2 & ESTIMACION 3 & ESTIMACION 4 \\
Constante & $63.98742^{*}$ & $55.23053^{*}$ & $54.08903^{*}$ & $59.00809^{*}$ \\
PIB per capita & $-0.0029134^{*}$ & $-0.0009528^{* *}$ & $-0.001067^{* *}$ & $-0,0007998$ \\
Tasa de desempleo & & $0.6158043^{*}$ & $0.6674778^{*}$ & $0.5363001^{*}$ \\
Gasto social \% del PIB & & $-0.3207236^{* *}$ & & $-0.305337^{*}$ \\
Gasto educacion \% del PIB & & $-0.7250804^{* * *}$ & \\
Indice de efectividad Gobierno & & $-1,335613$ & $-1,043702$ & $-0,714441$ \\
Recaudacion fiscal \% del PIB & & & 0,3045036 \\
R2 & 0,3721 & 0,4937 & 0,4822 & 0,5046 \\
Varianza de e & 2,4042642 & 2,1773284 & 2,2019044 & 2,2019044 \\
Varianza de u & 5,9725569 & 3,9901694 & 4,1413971 & 3,9737121 \\
Rho & 0,86054962 & 0,77055898 & 0,77961448 & 0,77194068 \\
\hline \\
estimacion por er r rores estandar agrupados
\end{tabular}

Tabla 3: Estimación GINI Variable dependiente

En la primera estimación se probó la variable de interés PIBpc, y esta efectivamente sustenta la hipótesis, ya que a un mayor crecimiento económico la desigualdad se reduce, es decir según esta estimación el incremento de una unidad en el PIBpc disminuye el coeficiente GINI en -0.0029134 y es significativa al 1\%, además se obtiene el signo esperado.

En la segunda estimación además de la variable PIBpc, se incluyeron las variables gasto social, tasa desempleo e IEG, y estas siguen sustentando la hipótesis de que a un mayor crecimiento 
económico la desigualdad disminuye, donde el aumento de una unidad en el PIBpc disminuye el coeficiente GINI en -0.0009528 y significativa al 5\%; en cuanto al gasto social el aumento de $1 \%$ disminuye el coeficiente GINI en -0.3207 y también es significativa pero al 5\%; el aumento de $1 \%$ en la tasa de desempleo aumenta el coeficiente GINI en 0.6158 y es significativa al $1 \%$; el IEG resultó no significativo pero se incluyó debido a que se busca reducir el sesgo de variable omitida. Todos los signos fueron los esperados.

En la tercera estimación se reemplazó el gasto social por gasto en educación, ya que esta variable es un factor importante para el desarrollo de un país; así mismo se sigue sustentando la hipótesis que a un mayor crecimiento económico la desigualdad disminuye, donde el aumento de una unidad en el PIBpc disminuye el coeficiente GINI en -0.0010, siendo significativa al 5\%; en cuanto al gasto en educación el aumento de $1 \%$ disminuye el coeficiente GINI en - 0.7250 siendo significativa al 10\%; el aumento de $1 \%$ en la tasa de desempleo aumenta el coeficiente GINI en 0.6674 siendo significativa al 1\%; así mismo el IEG resultó no significativo pero se la incluye por la misma razón anterior de reducir el sesgo de variable omitida. Todos los signos resultaron ser los esperados.

En la cuarta estimación se agregó la variable recaudación fiscal al modelo de la segunda estimación, lo cual ocasiona que el PIBpc se vuelva no significativo, aunque si obtuvo el signo esperado, además resultaron significativos tanto el gasto social como la tasa de desempleo y se obtuvo los signos esperados, el IEG y la recaudación fiscal no fueron significativos aunque también tuvieron el signo esperado. 
Evidencia de la disminución de la desigualdad y su relación con el crecimiento económico en América Latina

\section{Conclusiones.}

Este estudio se lo hizo exclusivamente para el período 2000-2012 debido a que la gran mayoría de investigaciones hacen un análisis del siglo anterior y hay pocos que lo realizan solamente para el presente siglo. Se ha evidenciado que algunos países han tenido incluso el menor índice de desigualdad en su historia, que por lo general ha sido en los últimos años del periodo estudiado, así mismo hay que destacar que el crecimiento económico promedio en la región durante el periodo analizado ha sido el mayor desde la denominada década perdida, estas razones fueron importantes para la elección del periodo estudiado para probar la hipótesis de la Curva Kuznets.

Se encontró evidencia que a un mayor crecimiento económico contribuye a disminuir la desigualdad, cabe mencionar y se debe recalcar que además de un importante crecimiento económico que obtuvo la región, han existido gobiernos con tendencia socialista que se han enfocado en la igualdad social con respecto a gobiernos anteriores, como es el caso de Ecuador, que ha mostrado importantes avances en el campo de la igualdad social, lo cual se considera una pieza fundamental para que se cumpla la hipótesis. Por esta razón se pudo evidenciar que el gasto social y el de educación han sido factores importantes, que han ido en un constante aumento en la mayoría de países, sirviendo como instrumento de los Gobiernos para reducir la desigualdad.

Se probó la variable recaudación fiscal en distintas estimaciones y con distintas medidas de la variable, pero no resultó significativa y tampoco contribuía al modelo como se esperaba, esto puede ser debido a que en realidad se desconoce a qué sectores el Estado destina los recursos, y qué consecuencias puede llegar a tener la distribución de ingresos vía fiscal, por esta razón no se puede concluir si en realidad esto contribuye a disminuir la desigualdad; resultaría necesario especificar el 
destino de los recursos para poder medir el efecto que se tendría sobre la desigualdad, pero es difícil obtener este tipo de información con lo cual esta variable estaría limitada para un correcto análisis.

Respecto al índice de efectividad gubernamental se la incluyó debido a que es necesario una medición que identifique qué tan efectivas han sido las políticas por parte de los distintos Gobiernos, esta no resultó significativa en ninguna estimación pero contribuía en gran manera al modelo, una razón de esto puede ser que no se especifica qué tipo de políticas son las evaluadas, incluso pueden estar alejadas del tema de igualdad social. Puede haber otras cuestiones que estén ligadas a este tema y podrían explicar mejor el modelo, pero no se las puede tomar en cuenta, ya que no hay la suficiente información disponible o no son medibles.

Una relevancia importante que es necesaria mencionar es que los Gobiernos de turno o posteriores, deben seguir preocupándose de disminuir la desigualdad, independientemente de la tendencia política a la que pertenezcan, ya que puede resultar problemático y contraproducente que cada cierto tiempo existan distintas tendencias en el poder y que hagan cambios estructurales que modifiquen todo lo logrado en cuestiones de desarrollo social. Ecuador al igual que otros Gobiernos de la región debe incentivar la igualdad entre los habitantes. Por esta razón, basados en la evidencia empírica del estudio, se debe continuar destinando recursos al gasto social, en donde una de las principales áreas es la de educación, siendo elementos relevantes para seguir en la lucha por una mayor igualdad social.

\section{Bibliografía.}

Adams, R. (2003). Economic Growth, Inequality and Poverty: Findings from a New Dataset. World Bank Policy Research Working Paper 2972.

Banco Mundial (2017). América Latina y el Caribe. Datos disponibles en: http://datos.bancomundial.org/region/america-latina-y-el-caribe?view=chart 
Evidencia de la disminución de la desigualdad y su relación con el crecimiento económico en América Latina

Bengoa, M. y Sánchez, R. (2001). "Crecimiento económico y desigualdad en los países latinoamericanos”. En: Información Comercial Española. No 790.

Comisión para América Latina y el Caribe, CEPAL. (2017). Estadísticas e Indicadores: http://estadisticas.cepal.org/cepalstat/WEB_CEPALSTAT/estadisticasIndicadores.asp?idioma=e

Dagdeviren, H., R. van der Hoeven y J. Weeks. (2005) "Redistribution dos matter: Growth and redistribution for poverty reduction", Prospects for pro-poor economic development, Nueva York, Oxford University Press-UNU-WIDER, pp. 125-153.

Janvry, A. y Saudolet, E. (1999). Growth, Poverty and Inequality in Latin America: A Causal Analysis 19701994. Inter-American Development Bank. Conference on Social Protection and Poverty.

Klasen, S. (2004) "In search of the Holy Grail: How to achieve pro-poor growth" Toward pro-poor policies: Aid, institutions and globalization, Washington D. C., Banco Mundial-Oxford University Press, pp. 63-93.

Kuznets, S. (1955) "Economic growth and income inequality", American Economic Review 45, 1, pp. 1-28.

Londoño, J. L. y M. Szequely. (1997) "Sorpresas distributivas después de una década de reformas: América Latina en los noventa", Pensamiento Iberoamericano. Revista de Economía Política, núm. especial, pp. 195-242.

Macías V., A. (2014). Crecimiento, desigualdad y pobreza: estado de la cuestión. Revista de Economía Institucional 16, 31, 2014, pp. 101-126.

Ocampo, J. A. (1998). Distribución del ingreso, pobreza y gasto social en América Latina. Revista de la CEPAL Vol. 68, pp 7-14.

Pearson, T. y Tabellini, G. (1994). “Is Inequality Harmful for Growth?”. En: The American Economic Review. Vol. 84, No 3.

Thornton, J. (2001) The Kuznets inverted-U hypothesis: panel data evidence from 96 countries, Applied Economics Letters, 8:1, 15-16.

Worldwide Governance Indicators (WGI) (2017). Efectividad Gubernamental. Datos disponibles en: http://info.worldbank.org/governance/wgi/\#home

2015; Perspectivas económicas de la OCDE: Proyecciones para países latinoamericanos; Edición I; QuitoEcuador; OCDE; Disponible en OCDE URL: https://www.oecd.org/eco/outlook/PerspectivasEcon\%C3\%B3micas-de-la-OCDE-Proyecciones-para-pa\%C3\%ADses-latinoamericanos.pdf; 20-042017

TALAVERA P. (1991). La crisis económica de América Latina. Sendai. Barcelona.

Palazuelos Manso Antonio; sin fecha; Introducción a la realidad económica latinoamericana; UCM; URL: https://www.ucm.es/data/cont/media/www/17360/untitled\%20folder/untitled\%20folder/lectura\% 204. pdf

S:F:; El desarrollo económico de la América Latina y algunos de sus principales problemas; Edición I; CEPAL; Disponible en REPOSITORIO.CEPAL URL: http://repositorio.cepal.org/bitstream/handle/11362/40010/prebisch_desarrollo_problemas.pdf; 20-042017 